\title{
Prototype Conduction Cooled Capture Solenoid Test Design and Plans
}

\author{
R. Carcagno, S. Feher, S. Hays, A. Hemmati, V. V. Kashikhin, M. J. Kim, L. Kokoska, M. J. Lamm, Y. Makida, \\ J. Nogiec, T. Ogitsu, D. F. Orris, T. Page, V. Poloubotko, R. Rabehl, W. Soyars, C. Sylvester, M. A. Tartaglia,
} A. Yamamoto, M. Yoshida

\begin{abstract}
Large aperture superconducting solenoid magnets are needed for the production and capture of pions, which decay to create intense muon beams in future experiments to search for direct muon to electron conversion. The COMET experiment in Japan, and the Mu2e experiment in the U.S. are jointly conducting research into the design of capture solenoid coils made from aluminum-stabilized NbTi superconductor that is cooled by conduction to a supply of liquid helium. A prototype coil of $1.3 \mathrm{~m}$ inner diameter, having 4 layers of 8 turns each, has been wound with pure aluminum interlayer fins for the conduction cooling. The test coil includes two types of welded splices, two film heaters for quench studies, and extensive instrumentation to evaluate strain, temperature profiles, and coil voltages. Details of the cryogenic conduction cooling scheme, test systems design, and test program plans will be discussed.
\end{abstract}

Index Terms-Superconducting Solenoid, Conduction Cooled, Detector Magnet, Prototype.

\section{INTRODUCTION}

$\mathrm{T}$ HE DESIGN of experiments to search for the very rare process of direct muon to electron conversion requires development of a new generation of large aperture, high field solenoid magnets, for muon production and transport, and detection of mono-energetic outgoing conversion electrons. While there are differences in the design details of these "intensity frontier" experiments being planned to operate at Fermilab [1] and J-PARC [2], there are many similarities and common magnet technology issues to solve, particularly with regard to the intense radiation environment in which the muon production, or "capture" solenoids will operate. Thus a joint U.S.-Japan R\&D effort has been ongoing for several years to fabricate prototype coils with appropriate radiation- and stressresistant superconductor and insulator materials, and test their mechanical, thermal, quench and magnetic performance. A detailed discussion of the motivating requirements for this

Manuscript received July 16, 2013. This work was supported in part by Fermi Research Alliance, LLC, under contract No. DE-AC02-07CH11359 with the U.S. Department of Energy, and in part by the Japan-U.S. cooperative program in High Energy Physics.

M. Tartaglia, R. Carcagno, S. Feher, S. Hays, A. Hemmati, V. Kashikhin, M. Kim, L. Kokoska, M. Lamm, J. Nogiec, D. Orris, T. Page, V. Poloubotko, R. Rabehl, W. Soyars, and C. Sylvester are with Fermi National Accelerator Laboratory, Batavia, IL 60510 USA (corresponding author phone: 630-8403890; fax: 630-840-8079; e-mail: tartaglia@ fnal.gov).

M. Yoshida, Y. Makida, T. Ogitsu, and A. Yamamoto are with the High Energy Accelerator Research Organization (KEK), Tsukuba, Ibaraki, 3120801 Japan (e-mail: makoto.yoshida@kek.jp). work was given, along with the fabrication and test results of an early model magnet, in a previous publication [3]. Subsequently, a new prototype capture solenoid coil was designed, fabricated at the Toshiba Corporation, instrumented at KEK, and shipped to Fermilab to be tested. Details of the "Toshiba Coil" fabrication and instrumentation are described in [4]. In this paper, we describe the design and construction status of the systems that will be used to support, cool, and power test this coil. An overview of the test program to evaluate the performance, in a non-radioactive environment, will also be discussed.

\section{Coil Construction Overview}

The Toshiba Coil design and construction details are reported in [4], but an overview of the salient features is provided here as they relate to evaluating the magnet baseline performance (absent the nuclear radiation environment). The coil consists of 4 layers with 8 turns per layer of $15.3 \mathrm{~mm} \mathrm{x}$ $5.0 \mathrm{~mm}$ (insulated) aluminum-stabilized $\mathrm{Cu} / \mathrm{NbTi}$ Rutherford cable manufactured by Hitachi, wound the hard-way bend with an inner radius of $650 \mathrm{~mm}$. Pure aluminum (5-N grade) $2 \mathrm{~mm}$ thick x $50 \mathrm{~mm}$ wide strips were placed between each layer and adjacent to the inner and outer perimeters, covering about $25 \%$ of the surfaces. The coil was wet-wound with a radiation-resistant BT based resin and cured 5 hours at $180{ }^{\circ} \mathrm{C}$.

Two welded joints were incorporated into the coil: one between the $2^{\text {nd }}$ and $3^{\text {rd }}$ layers, and one between turns on the outermost layer. Two film heaters are embedded into the same azimuthal region of the coil: one covers all turns between layers 2 and 3, the other spot heater is between two turns of the outer layer; these will be capable of generating $38 \mathrm{~W}$ and $12 \mathrm{~W}$ of heat, respectively, for studies of thermal resistances and raising coil temperatures for quench studies. The heaters are located midway between two sets of cooling fins.

The coil was instrumented with 41 voltage taps for characterization of quench development and joint resistance, as well as to provide half-coil voltage signals for quench detection. These taps are primarily located on the upper- and lower-most turns of each layer spaced at four azimuthal positions around the coil, and also surrounding the outer layer heater. Each tap was installed by grinding off the resin locally, then drilling and installing a self-tapping M1 screw into the aluminum stabilizer.

The coil is mechanically supported by a structure of 12 clamps made from G10 plates above and below the coil that 
are bolted together; the plates are interconnected by additional G10 bars at the inner and outer radii, both above and below the coil. A total of 16 strain gauges were mounted to the coil, at 4 inner radius and 4 outer radius mid-plane points, to monitor both axial and circumferential coil stresses during the test. A photograph of the coil is shown in Fig. 1.

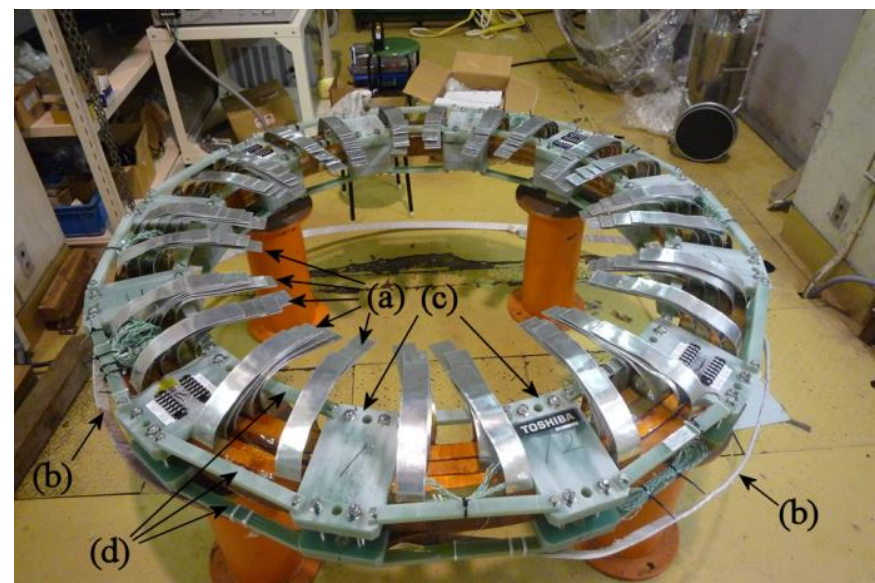

Fig. 1. The instrumented Toshiba Coil at KEK prior to shipment to Fermilab for testing. Aluminum conduction cooling fins (a) are seen on top, and insulated superconductor leads (b) extend below the coil which is supported by G10 plates (c) and G10 bars (d).

\section{Solenoid Test Facility}

A new facility (SolTF) for testing large aperture conduction-cooled solenoids has been under development at Fermilab [5], and is currently at the stage of cryogenic and power system commissioning. The need for such a facility was recognized several years ago as a rise in the development of large solenoids for high energy accelerator (MICE) and particle physics (Mu2e) experiments was foreseen. The key component allowing the very rapid construction of this large new test stand was acquisition of an existing, available, large volume SMES test cryostat from FSU in October 2011.

Significant modifications to the cryostat, and construction of sophisticated systems for control and monitoring, powering, quench detection and data acquisition have since been made; it was installed in the Fermilab Central Helium Liquifier (CHL) building which provides cryogenic services for the test stand. The inaugural test subject is the first MICE Coupling Coil (CC); some modifications to the stand for high current operation will then be made before testing of the Toshiba Coil can begin.

\section{A. Conduction Cooling Scheme}

The SolTF has the capability of mixing liquid helium with warm helium gas, to regulate the helium temperature for a controlled cool down, or maintaining a fixed temperature of a test subject. The maximum liquid helium flow rate is $10 \mathrm{~g} / \mathrm{s}$, at a minimum temperature of $4.6 \mathrm{~K}$. To cool the $208 \mathrm{~kg}$ Toshiba Coil, the 2-phase helium will be routed through a horizontal cooling circuit around the circumference of the coil; the cooling scheme, then, is to clamp the aluminum fins to the aluminum cooling channel. The cooling channel will be 1.125 inch square 6061 aluminum tubing, with a 0.875 in. diameter hole for helium flow. Fig. 2 shows a 3D model image of the cooling channel below the coil; the channel itself will be mechanically secured to the coil G10 supports with brackets. An important design consideration was to break the cooling loop with an electrical insulator, in order to prevent large forces on the channel and cooling fins following a quench, due to transformer coupling to the coil and the expected large $\mathrm{dI} / \mathrm{dt}$ (combined with very low cooling channel resistance).

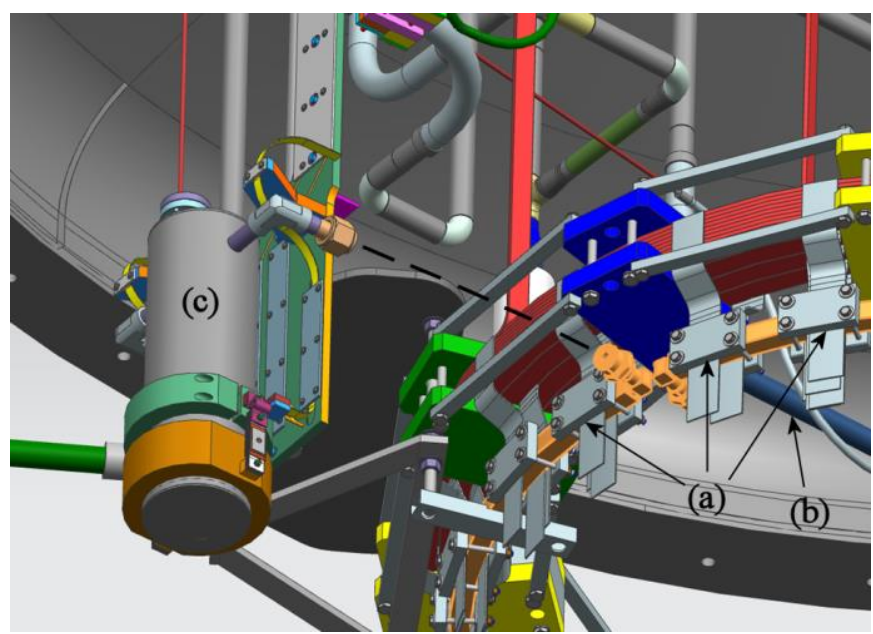

Fig. 2. 3D model close up view of the cooling channel design for test of the Toshiba Coil, showing fins clamped to the helium channel (a). The helium supply (b) connects from the right side; connection to the helium pot (c) on the left is not shown (dashed line).

After flowing around the channel, the helium then spills into a helium pot which collects liquid. A heater in the pot is used to maintain the pot liquid level and boil liquid off for return to the cryogenic plant as gas. The helium pot is useful to determine that the entire circuit is cooled by liquid helium, and can provide a $\sim 4.6 \mathrm{~K}$ temperature sink for conduction cooling of solenoid current leads (needed for MICE).

\section{B. Coil Supports}

The coil and cooling features will be suspended from the cryostat top plate assembly, as shown in Fig. 3. Support rods connect to plates that secure the coil via bolts through holes in the coil G10 clamps at three locations. To intercept the conducted heat through these supports, thermal straps will be anchored to the nearby helium cooling channel. Lateral forces from magnetic interactions with ferromagnetic materials in the vicinity are reacted via lateral support rods that contact the vacuum vessel walls. Detailed estimates of the forces and torques on the coil using Opera3D are in progress. However this was done for the case of the MICE CC magnet at the highest operating field to be $3000 \mathrm{~N}$ (transverse) and 8000 $\mathrm{Nm}$. The MICE CC peak field on axis is $2.6 \mathrm{~T}$, whereas the Toshiba Coil peak field will be $0.6 \mathrm{~T}$ at most (at $20 \mathrm{kA}$ ). Thus scaling by the ratio of the squared field strengths gives a preliminary estimate of $160 \mathrm{~N}$ and $424 \mathrm{Nm}$ for the Toshiba Coil maximum transverse force and torque.

\section{Thermometry}

Approximately 30 temperature sensors will be mounted to the coil surface and other components at Fermilab, in a variety of locations to measure the actual performance as test 
conditions are varied, and to estimate heat loads and thermal resistances in conjunction with finite element model simulations. Note that it is not planned to remove any resin to attach sensors directly to the aluminum stabilizer on the coil.

The SolTF cryostat does not have a thermal shield to intercept $300 \mathrm{~K}$ radiated heat, and relies solely on good insulating vacuum and multi-layer insulation around the entire inner assembly, and also around the coil and cooling features. An $80 \mathrm{~K}$ thermal intercept may be considered once heat loads are understood in the MICE CC test. The estimate of conducted heat is about $1.3 \mathrm{~W}$ through each vertical support, and $0.3 \mathrm{~W}$ through each lateral support, most of which will be intercepted by the thermal straps attached to the cooling channel (see Fig. 3). Sensors will be placed on the coil and cooling channels in 8 locations near, and on, one of the supports, to directly measure the temperature profile in that region of high heat flux.

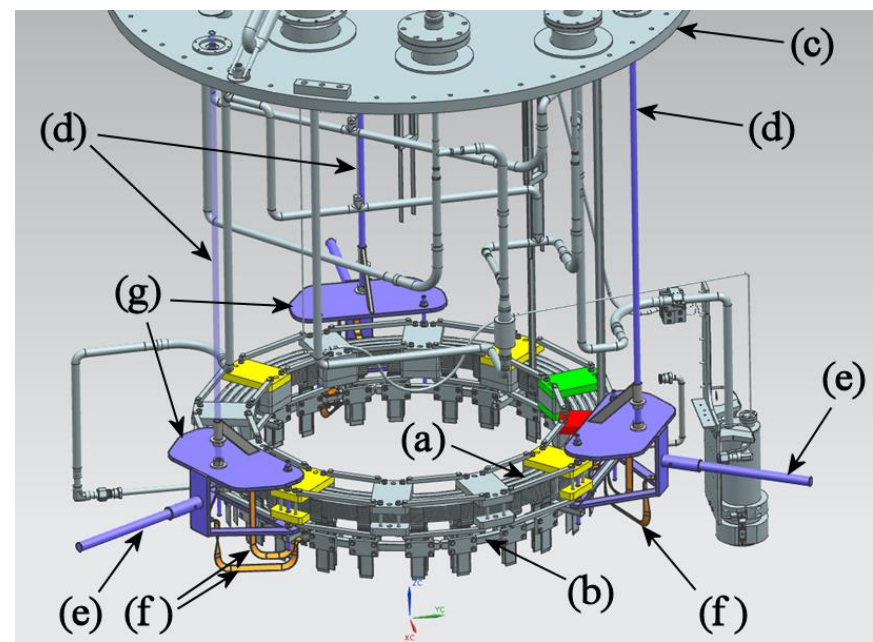

Fig. 3. 3D model view of the coil (a) and cooling channel (b) suspended from the top plate (c) by vertical support rods (d) and constrained by lateral support rods (e). Thermal straps (f) between the support plates (g) and cooling channel (b) are shown.

The pattern of thermal sensors in the support region will mirror another set that are located in a typical section of the coil that is far from the supports and heaters. Both sets will measure a baseline temperature on the cooling channel surface, on cooling fins just above the coil, and around the coil surface in the region mid-way between sets of cooling fins. In the heater region, the sensors will be distributed around the coil top, bottom, sides, and possibly on cooling fins, to monitor the temperature profile during heater studies. Sensors will be positioned on the superconducting leads (on the aluminum stabilizer) near the coil, and also near the LTS end of the HTS current leads.

\section{Thermal Model}

Based upon the cooling channel and mechanical support engineering designs, a thermal model has been built to estimate thermal time constants and temperature distributions on the coil and other components, given various assumptions about environmental and operating conditions. Convective heat transfer between the helium and cooling channel is assumed. Some uncertainty exists for the thermal resistance associated with the resin between coil turns and layers; this is assumed to be the same as Kapton. The overall heat load estimate to the $4.6 \mathrm{~K}$ helium system is approximately $6.8 \mathrm{~W}$. Estimated time to cool the coil from room temperature to $4.6 \mathrm{~K}$, at $10 \mathrm{~g} / \mathrm{s}$ flow rate, is 40 hours. Fig. 4 shows a simulated temperature distribution of the coil assuming a $4 \mathrm{~g} / \mathrm{s}$ helium flow rate; the highest temperatures are near the supports. Fig. 5 shows the predicted temperature profile under these same conditions in the heater region, but after both heaters have been powered $(40 \mathrm{~W})$ for less than 10 minutes.

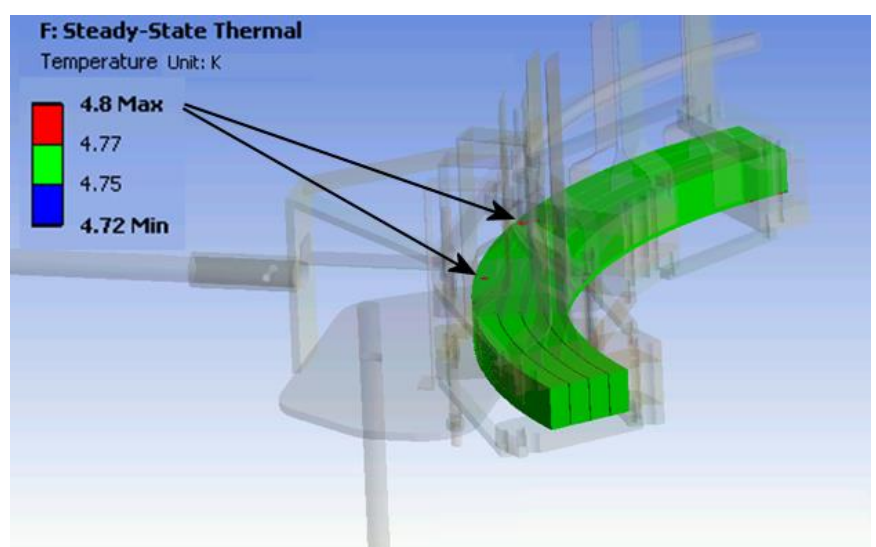

Fig. 4. ANSYS thermal model predicted temperature distribution around the coil for nominal $6.8 \mathrm{~W}$ heat load and $10 \mathrm{~g} / \mathrm{s}$ helium flow rate at $4.6 \mathrm{~K}$.

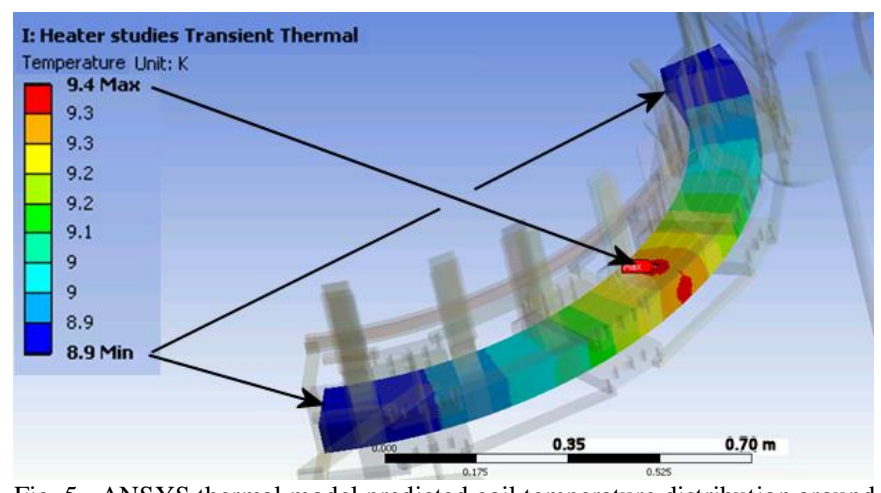

Fig. 5. ANSYS thermal model predicted coil temperature distribution around the heaters with $50 \mathrm{~W}$ total heater power at nominal $4 \mathrm{~g} / \mathrm{s}$ helium flow.

The Hitachi superconductor has a very high critical current, $40 \mathrm{kA}$ at $4.2 \mathrm{~K}$ and $5 \mathrm{~T}$ field. The estimated critical temperature of this conductor in the Toshiba Coil is shown in Table I at several operating currents. The power system and current leads are being designed for a maximum current of $20 \mathrm{kA}$. We envision mapping out the critical surface by varying the coil temperature, either locally with film heaters, or globally by controlling the helium liquid temperature or lowering the helium flow rate.

\section{TABLE I}

TOSHIBA COIL CRITICAL TEMPERATURE

\begin{tabular}{cc}
\hline \hline Current $[\mathrm{kA}]$ & Critical Temperature $[\mathrm{K}]$ \\
\hline 4 & 8.3 \\
8 & 7.2 \\
16 & 5.6 \\
\hline
\end{tabular}

\section{E. HTS and LTS Current Leads}

The capture solenoid in the mu2e experiment will operate at a current of nearly $10 \mathrm{kA}$, and plans are to utilize existing HTS 
current leads that were developed for the Tevatron. These leads have been previously qualified to operate at $10 \mathrm{kA}$ for the BTev experiment [6]. At present they are being requalified for use in a conduction-cooled, rather than bathcooled, environment. This work is in conjunction with $R \& D$ studies of aluminum-stabilized conductor welded joints [7], which is nearing completion. In this approach aluminum tubing is welded to the aluminum stabilizer, and a flow of helium in the tubing cools the cable by conduction.

Two pairs of these HTS leads will be installed in existing flanged ports on the SolTF top plate to allow powering the Toshiba Coil up to $20 \mathrm{kA}$. The HTS leads require liquid nitrogen (LN2) to maintain the $80 \mathrm{~K}$ temperature at the copper upper section joint. Existing (Tevatron) LN2 reservoirs for this will be supported off the top plate, and the detailed mechanical design is nearing completion.

Design of the HTS/LTS joint will follow from the design in [7], where a soldered joint is made to the NbTi conductor from the etched end of an aluminum-stabilized cable. Connection of this cable to the Toshiba Coil leads is still under discussion; it may be another NbTi-NbTi soldered joint (stabilized with copper block), or it could be a welded splice. Also, still to be determined is the helium flow scheme for cooling these LTS and HTS leads.

\section{F. Power and Protection System}

A switcher-cell power system for the Toshiba Coil has been assembled from modular low voltage, high current components and testing is in an advanced stage. Each module can provide up to $15 \mathrm{kA}$ at up to $1.24 \mathrm{~V}$, and two modules can be connected in series to allow $2.48 \mathrm{~V}$ at $15 \mathrm{kA}$, or up to 30 $\mathrm{kA}$ at $1.24 \mathrm{~V}$. Allowing $0.1 \mathrm{~V}$ for ramping the very low inductance $(2.7 \mathrm{mH})$ Toshiba Coil, to reach $20 \mathrm{kA}$ will require the power bus resistance to be below $61.5 \mu \Omega$. Thus, to achieve $20 \mathrm{kA}$ will require care to keep the bus short and resistance low. Water-cooled round copper bus of $3.4 \Omega / \mathrm{ft}$ resistance will be used, and will be split into parallel paths for connection to each HTS leads pair, which will ensure current sharing between the HTS leads.

Because of the low inductance, and relatively low stored energy $(0.54 \mathrm{MJ}$ at $20 \mathrm{kA})$, an energy extraction switch and resistor will not be needed. Once the coil develops any significant resistive voltage $(\sim 0.5 \mathrm{~V})$, a Shottky diode will conduct and the power system will ramp down with the V/L time constant of the diode: at its peak the current will fall at a rate of $(1.455 \mathrm{~V}) / \mathrm{L}$.

Studies of the coil quench development and hot spot temperature are under way; preliminary indications suggest the peak temperature will be below about $100 \mathrm{~K}$ for a $20 \mathrm{kA}$ quench. So, it may be that active quench detection to turn off the power supply is not required in this case. However, a quench detection and data logging system will be used for characterizing quench locations and development. This information will be valuable for developing protection techniques for the actual experiment solenoids.

\section{Test Program Overview}

The Toshiba Coil design is most similar to that of the COMET capture solenoid, in cross section and conductor properties, which is being designed to operate at $8 \mathrm{kA}$ in a $5 \mathrm{~T}$ self-field at $4.2 \mathrm{~K}$ with large operating margin; the Mu2e capture solenoid is larger in diameter and will operate near $10 \mathrm{kA}$. The limited number of turns in the Toshiba Coil will limit the self-field at $8 \mathrm{kA}$ to approximately $1 \mathrm{~T}$, so the test program will explore the force and conductor limits by operating at currents up to $20 \mathrm{kA}$, and varying the coil temperature through control of input helium temperature, flow, and using inter-layer heaters.

It is important input for the final magnet designs to understand the efficiency of conduction cooling by the use of interlayer cooling fins that conduct heat from the coils.

For the Mu2e experiment, high current operation requires the use of HTS current leads. In this test, the leads intended for this purpose will be operated under conditions similar to those planned for the actual experiment. Furthermore, experience will be gained with methods of cooling long lengths of aluminum-stabilized superconducting leads.

Both COMET and Mu2e systems consist of several large and magnetically coupled solenoids, within which there may be long lengths of superconducting bus. This prototype magnet and test setup will allow testing of quench detection methods for both the coil and the superconducting leads.

Aluminum-stabilized $\mathrm{NbTi}$ cable joints pose special challenges, and many splices are anticipated in these large solenoid systems. These may be welded joints, or soldered NbTi-NbTi after removing the stabilizer. Thus, gaining experience with splices and their properties will be valuable input for the final design and fabrication of these solenoids.

\section{CONCLUSION}

A prototype capture solenoid has been fabricated and instrumented to study a variety of performance parameters, to gain information relevant to the final design of magnets and related systems for muon conversion experiments planned in the U.S. and Japan. The design and capability of the test stand subsystems was described, and in general the status is advanced. Expectations are to complete testing of the Toshiba Coil by the end of this calendar year.

\section{REFERENCES}

[1] http://mu2e.fnal.gov/

[2] http://comet.phys.sci.osakau.ac.jp:8080/comet/internal/publications/comet-cdr-v1.0.pdf.

[3] N. Andreev, et al., IEEE Trans. Appl. Supercond. 22(2012)6001804.

[4] M. Yoshida, et al., "Development of a Radiation Resistant Superconducting Solenoid Magnet for mu-e Conversion Experiments," IEEE Trans. Appl. Supercond. 23(2013)4101404.

[5] R. Rabehl, R. Carcagno, J. Nogiec, D. Orris, W. Soyars, C. Sylvester, "A Cryogenic Test Stand for Large Superconducting Solenoid Magnets," submitted to the 2013 CEC/ICMC conference.

[6] S. Feher, et al., "HTS Power Leads for the BTEV Interaction Region," Proceedings of the 2005 Particle Accelerator Conference.

[7] S. Feher, et al., "Splice Studies for the Mu2e Solenoid System," submission to this conference. 